\title{
Author Correction: Chern insulators, van Hove singularities and topological flat bands in magic-angle twisted bilayer graphene
}

\author{
Shuang Wu, Zhenyuan Zhang, K. Watanabe (D), T. Taniguchi and Eva Y. Andrei (D)
}

Correction to: Nature Materials https://doi.org/10.1038/s41563-020-00911-2, published online 15 February 2021.

In the version of this Article originally published, some of the Chern numbers had incorrect signs.

In Fig. 3b, the top-left label was incorrect as $C=-1$ and the top-right label was incorrect as $C=+1$; they should have been $C=+1$ and $C=-1$, respectively. In the Fig. $3 \mathrm{~b}$ caption, the text ' $C=-1$ (yellow) and $C=+1$ (green)' should have read ' $C=+1$ (yellow) and $C=-1$ (green)'. In addition, the real-space current density distribution in Fig. 3a (bottom) has been updated.

In the Fig. 4 caption the Chern numbers were incorrectly given in two instances as $C=-2,-1,+2,+3$; in both instances they should have been $C=+2,+1,-2,-3$. In addition, in the caption for Fig. $4 \mathrm{c}, \mathrm{d}$ the expression for the Hall plateau values was incorrect as $R_{x y}=\frac{1}{C} \frac{h}{e^{2}}$; it should have been $R_{x y}=-\frac{1}{C} \frac{h}{e^{2}}$.

Finally, in the caption for Extended Data Fig. 8 the Chern number was given incorrectly in two instances as $C=1$; it should have been $C=-1$ in both instances.

These errors have now been corrected.

Published online: 1 June 2021

https://doi.org/10.1038/s41563-021-00997-2

(c) The Author(s), under exclusive licence to Springer Nature Limited 2021

\section{Publisher Correction: Mixed matrix formulations with MOF molecular sieving for key energy-intensive separations}

Gongping Liu, Valeriya Chernikova, Yang Liu, Kuang Zhang, Youssef Belmabkhout, Osama Shekhah (D), Chen Zhang, Shouliang Yi, Mohamed Eddaoudi (D) and William J. Koros

Correction to: Nature Materials https://doi.org/10.1038/s41563-017-0013-1, published online 12 February 2018.

In the version of this Article originally published, in the $y$-axis label in Fig. 3a the unit was incorrect as ' $10^{-2} \mathrm{~cm}^{3}\left(\mathrm{STP} \mathrm{cm}^{-1} \mathrm{cmHg}^{-1}\right.$; it should have been ' $10^{-2} \mathrm{~cm}^{3}(\mathrm{STP}) \mathrm{cm}^{-3} \mathrm{cmHg}^{-1}$. In addition, in the schematic in the lower-right corner of Fig. $3 \mathrm{e}$ the label ' $<d_{i} \mathrm{C}_{4}$ ' was incorrect; it should have been ' $>d_{i \mathrm{C}_{4}}$ '.

Published online: 27 May 2021

https://doi.org/10.1038/s41563-021-01007-1

(c) The Author(s), under exclusive licence to Springer Nature Limited 2021 